\title{
Treadmill Low-Intensity Training Mediates Fibrosis and Maintains the Function of Dystrophic Muscle: Understanding the Effects Over Time
}

\author{
A. Ferreira Lemes de Morais', P. Avelino Ferreira Pinto ${ }^{3}$, A. Nobre Evangelista', \\ A. P. Santos ${ }^{1}$, A. S. Dias Machado ${ }^{2}$, T. Peixoto Gaiad ${ }^{1}$ \\ 1 Department of Physical therapy, Faculty of Health and Basic Science, Universidade Federal dos Vales do \\ Jequitinhonha e Mucuri - UFVJM, Brazil \\ 2 School of Medicine, Universidade Federal dos Vales do Jequitinhonha e Mucuri - UFVJM, Brazil \\ 3 Department of Surgery, School of Veterinary Medicine and Animal Science, Universidade de São Paulo, São \\ Paulo, Brazil
}

\section{CORRESPONDING AUTHOR: \\ Thais Peixoto Gaiad \\ Universidade Federal dos Vales do \\ Jequitinhonha e Mucuri, UFVJM \\ Campus JK - Diamantina/MG Rodovia \\ MGT 367 \\ 5000 Alto da Jacuba, Brazil \\ E-mail: thaispgm@gmail.com}

DOI:

10.32098/mltj.04.2020.20

LEVEL OF EVIDENCE: $2 B$

\begin{abstract}
SUMMARY
Background. Dystrophic skeletal muscle undergoes fast degeneration and therapeutic exercise is controversial in this condition. The aim of this study was investigate the effects of a low-intensity training protocol on muscle fibrosis and function of the $m d x$ model.

Methods. $M d x$ male animals with 8 weeks of age were $(\mathrm{T} 0 ; \mathrm{n}=8)$ underwent a protocol of eight weeks at a horizontal treadmill ( $9 \mathrm{~m} / \mathrm{min}, 3 \mathrm{x} /$ week, $30 \mathrm{~min} / \mathrm{day}) . M d x$ animals were randomly allocated at the Trained group $(m d x-\mathrm{T}, \mathrm{n}=8)$ or sedentary $(m d x-\mathrm{NT}$, $\mathrm{n}=8$ ). In vivo functional measurements of strength and performance, immunolocalization of TGF- $\beta 1$ and histomorphometry of intramuscular collagen fibers were assessed over time of protocol (T0, T4, T8) on Tibial Anterior (TA) and Soleus (SOL).

Results. Strength decreased after 4 weeks of training (T4) and was regained at T8 at $m d x$-T. The percentage of intramuscular collagen fibers area decreased at SOL muscle of $m d x$-T at T4 when compared to T0 $(\mathrm{p}=0,025)$ and TA of $m d x$-T had a lesser area at T8 when compared to TA of $m d x$-NT group $(\mathrm{p}=0,002)$. TGF- $\beta 1$ was observed at the sarcoplasm of TA and SOL muscles of $m d x$-NT group, with an age-dependent change. Conclusions. Treadmill low-intensity training provoked dystrophic skeletal muscle adaptation of fibrosis and maintained grip strength of the $m d x$ model.
\end{abstract}

\section{KEY WORDS}

Duchenne muscular dystrophy; mdx model; low-intensity training; treadmill; fibrosis; $T G F-\beta 1$.

\section{BACKGROUND}

Duchenne Muscular Dystrophy (DMD) is the most common muscular dystrophy that affects 1:3500 boys due to an $\mathrm{x}$ linked disorder with genetic inheritance. Mutations in the dystrophin gene lead to protein expression deficiency resulting in muscular degeneration, necrosis and atrophy (1). Dystrophin is typically expressed on skeletal, smooth and cardiac muscles and on the brain but its absence on DMD is particularly observed on skeletal and cardiac muscles (2). The muscle damage is constant and outpaces the muscles normal ability for self-regeneration through its resident population of adult stem cells (satellite cells). In dystrophin's absence, there is increased cellular membrane damage (even under light muscle tension), constant necrotic inflammation, and satellite cell exhaustion. Eventually, muscle cells are replaced with nonfunctional fibrotic collagen build up and adipose tissue accumulation (3) inflammatory cells that infiltrate at the necrosis place are a source of transforming growth factor beta (TGF- $\beta$ ). TGF- $\beta$ is a multifunctional cytokine that acts in different cellular types. Its role on the regulation of skeletal muscle inflammatory response, inhibition of regeneration, regulation of the extra- 
cellular matrix remodeling and fibrosis promotion is well established on literature (3), and also is positively regulated on $m d x$ muscle (4).

Skeletal muscle fibrosis increases the area of Extracellular Matrix (ECM) on muscle sections and, as a result of the high deposition of collagen fibers, increases of muscle stiffness and contractibility; it limits muscle function and mobility. Muscle fibrosis also limits the amount of available muscle tissue to therapy and repair (4).

Important events of DMD natural history were clarified by preclinical studies using the $m d x$ model. They include the sarcolemma mechanical fragility (5), aberrant calcium homeostasis (6), mitochondrial distress, imbalanced oxidative stress (7) and chronic inflammation (8).

The endurance training was proposed to enhance muscle strength and avoid contractures on boys with DMD, but the impact and beneficial mechanisms due to the endurance exercise regimen need more research $(9,10)$ once the studies with $m d x$ model produced inconsistent results.

High intensity training, as a horizontal treadmill running at high speeds of $12 \mathrm{~m} / \mathrm{min}$, has been suggested to exacerbate muscular dystrophy (11). In the other hand, voluntary exercise as swimming and voluntary wheel running seems to delay the disease progression $(10,12,13)$. However, an exactly dose of these therapeutic exercise considered as of low intensity has not been determined yet. According to Kostek and Gordon (12) it is unknown how much stress is necessary to induce the positive effects of exercise without exacerbate the disease.
This study investigated the effects of chronic low-intensity training on function and regeneration of skeletal muscle of the $m d x$ model over time.

\section{MATERIALS AND METHODS}

Forty males dystrophic $m d x$ mice (C57BL/10ScSn- Dmd $m$ $d x / \mathrm{J}$ ) acquired from UniABC, São Paulo, Brazil (CQB172/02) were studied. Animals were maintained in cages on a $12 \mathrm{~h}$ day $/ 12 \mathrm{~h}$ dark inverted cycle with ambient temperature controlled at $22{ }^{\circ} \mathrm{C}$ and supplied with food and water ad libitum. Thirty-two $m d x$ animals were randomly assigned into two groups: Trained $(m d x-\mathrm{T}, \mathrm{n}=16)$ or No-Trained $(m d x-\mathrm{NT}, \mathrm{n}=16)$ group. The other eight animals were control $(n=8)$ of the initial time T0. This research was approved by the Ethics Committee on Animal Use of the Universidade Federal dos Vales do Jequitinhonha e Mucuri (CEUA/ UFVJM), protocol $n^{\circ} 025 / 15$ and meets the ethical standards of the Muscles, Ligaments and Tendons Journal (14).

\section{Design}

Training protocol began after the first assessment (T0). One group had trained for 4 weeks - T4: Short protocol $(m d x-\mathrm{T}=8$ and $m d x-\mathrm{NT}=8)$ and another one had trained for 8 weeks - T8: Long protocol $(m d x-\mathrm{T}=8$ and $m d x-\mathrm{NT}=8)$ (figure 1). T0 was determined at the time dystrophic animals had 8 weeks of age. The age of 8 to 12 weeks is considered a morphological stable phase of the disease to $m d x$ model

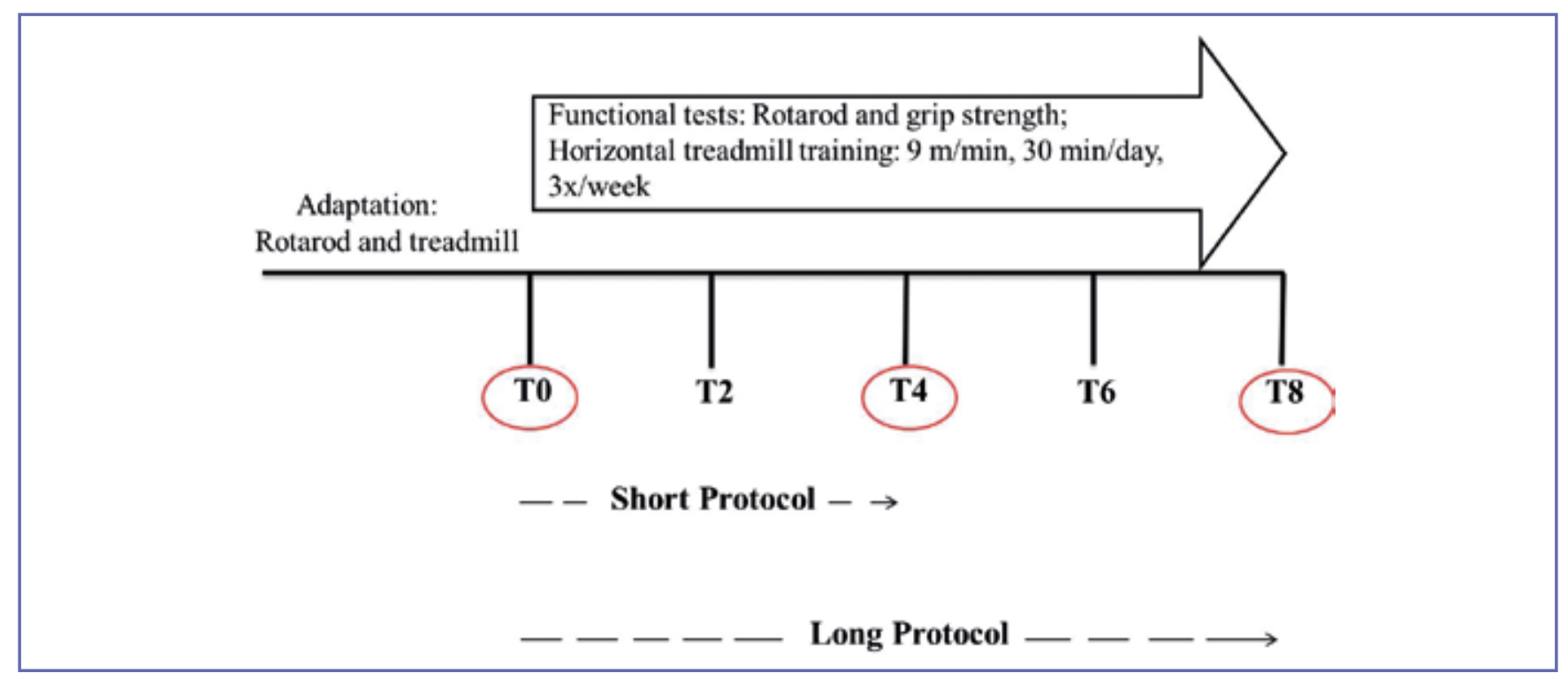

Figure 1. Experimental design: Times of assessment and collection of biological collection. Functional measurements of $\mathrm{mdx}-\mathrm{T}$ and mdx-NT: Rotarod and Grip Strength at T0, T2, T4, T6 e T8; Biological collection at T0, T4 e T8. 
once animals have already suffered an important cycle of degeneration/regeneration (15).

\section{Training protocol}

After an adaptation protocol (table I), $m d x$-T group were stimulated to run at a motorized treadmill (EP 131; Insight ${ }^{\circledR}$, Brazil) at $9 \mathrm{~m} / \mathrm{min}$. The speed was controlled at $9 \mathrm{~m} / \mathrm{min}$ so the protocol was considered of low intensity according to previously described protocols specific to this animal model (16). The protocol consisted of $30 \mathrm{~min} /$ day, $3 \mathrm{x} /$ week during 4 weeks (Short protocol -T4) or 8 weeks (Long protocol T8). Animals of $m d x$-NT group were placed at the horizontal treadmill with speed at $0 \mathrm{~m} /, 3 \mathrm{x} /$ week during 4 weeks aiming to be exposed to the same environmental conditions.

\section{Functional tests}

Grip Strength and Rotarod were used as functional measurements. They were assessed each 2 weeks of protocol over time (T0, T2, T4, T6 and T8) (figure 1). Every moment of functional measurements animals were weighted at a semi-analytic balance (UX- $420 \mathrm{H} 0,001 \mathrm{~g}$ precision) to follow animals well-being and also to further use to normalization the values of grip strength avoiding the influence of weight on this variable as described by Van Putten et al. (17).

\section{Rotarod}

Performance at Rotarod was used to assess coordination, balance, muscle strength and condition over time of protocol (17). An automated device was used (MP13977, Insight $^{\circledR}$, Brazil). After adaptation at the device (table II), the mice were placed in the rotarod tube as it rotated at a slow and steady speed of $5 \mathrm{rpm}$. The running started as soon as all the mice were in position. The tube speed accelerated from 5 to $37 \mathrm{rpm}$ and maintained that speed. The running time was stopped automatically when mice dropped from the tube, as this activated the time bar positioned below the tube, but the animals were repositioned immediately. The test session was terminated for mice capable of running for

Table I. Adaptation protocol of $\mathrm{mdx}-\mathrm{T}$ group at the treadmill.

\begin{tabular}{ll}
\hline Time & Speed \\
\hline $4 \mathrm{~min}$ & $2 \mathrm{~m} / \mathrm{min}$ \\
\hline $15 \mathrm{~min}$ & $4 \mathrm{~m} / \mathrm{min}$ \\
\hline $30 \mathrm{~min}$ & $6 \mathrm{~m} / \mathrm{min}$ \\
\hline $15 \mathrm{~min}$ & $9 \mathrm{~m} / \mathrm{min}$ \\
\hline $30 \mathrm{~min}$ & $9 \mathrm{~m} / \mathrm{min}$ \\
\hline
\end{tabular}

*Adaptation protocol started at the week before the training protocol where $\mathrm{mdx}$ animals were 7 weeks of age and lasted 5 days long.
Table II. Adaptation protocol to Rotarod.

\begin{tabular}{ll}
\hline Time & Rotarod speed \\
\hline $250 \mathrm{sec}$ & $16 \mathrm{rpm}$ \\
\hline $250 \mathrm{sec}$ & $25 \mathrm{rpm}$ \\
\hline $500 \mathrm{sec}$ & $37 \mathrm{rpm}$ \\
\hline
\end{tabular}

*Rotarod adaptation started at the week before the training protocol where $\mathrm{mdx}$ animals were 7 weeks of age and lasted 3 days long.

500 seconds. The mice were given a maximum of two more attempts, which allowed them to improve their execution time when they fell earlier. The maximum execution time (i.e. the longest of the tests) was used for further analysis (17).

\section{Grip Strength test}

The forelimb grip strength was performed with the Grip Strength Meter (PanLAb ${ }^{\circledR}$, Brazil). Animals were suspended by the tail above the grid and after they grasped, they were pulled backwards. The value of maximal force was registered by the Grip Strength Meter. Each animal was tested five times with one minute interval between trials. The three highest values were averaged to calculate the absolute strength, which was divided by the corporal mass in grams. All measurements were made by the same blinded investigator to avoid bias.

\section{Muscle tissue analysis}

Animals were euthanized during the protocol period: $\mathrm{n}=8$ at T0, $\mathrm{n}=16$ at $\mathrm{T} 4$ and $\mathrm{n}=16$ at $\mathrm{T} 8$ according to CEUA protocol $n^{\circ} 025 / 15$ seventy-two hours after the final exercise session. All animals were subjected to an overdose of ketamine hydrochloride $(200 \mathrm{mg} / \mathrm{kg})$ and xylazine hydrochloride $(20 \mathrm{mg} / \mathrm{kg})$, via intraperitoneal injection. Tibial Anterior (TA) and Soleus (SOL) muscle were collected and fixed in paraformaldehyde solution at $4 \%$ during $24 \mathrm{~h}$ and further tranfered to phosphate buffer. They were treated with increasing ethanol concentrations ( 70 to $100 \%$ ) to dehydrate and with xylene to clear. The samples were then embedded in paraffin (Ervplast ${ }^{\circledR}$ ) and sections of $5 \mathrm{~mm}$ in thickness were obtained. The cross-sections were ovendried $\left(60^{\circ} \mathrm{C}\right)$ at a horizontal position for better adhesion of the cuts. After deparaffinization protocol, the sections were stained using Hematoxylin-Eosin (HE) according to conventional histological procedures to identify histopathological features. Histochemical reaction using Picrossirius red, a combination of Sirius red F3BA (Sigma-Aldrich, Color Index 35780) dissolved in a saturated picric acid solution, was used in order to distinguish collagen from the skeletal muscle fibers. 


\section{Intramuscular collagen fiber quantification}

Slides reacted with picrossirius red were analyzed under polarized light in 400x. Photomicrographs of $\sim 20$ sequential images of each animal studied were performed to carry out the analysis of the whole section transverse muscle (18) totalizing an analysis of an area of 2000 to 3000 fibers/ animal. The amount of deposition of the collagen fibers was calculated by the percentage of the area of collagen fibers in relation to the total area $\left(57248.52 \mu \mathrm{m}^{2}\right)$ of each image, through binary analysis (black/white) and expressed in micrometers using Image ${ }^{\circledR}$ software: "Process" > "Binary" > "Make Binary" > "Analyze" > "Set Measurements" > selected "Area" + Area fraction" > "Analyze" > "Measure".

\section{Immunohistochemical analysis (IHC)}

Primary polyclonal antibodies against TGF $\beta-1$ (anti-human) (StressMarq Biosciences ${ }^{\circledR}$ ), 1:750 were applied on TA and SOL muscle sections. Sections were immersed in citric acid solution at $0.01 \mathrm{M}, \mathrm{pH} 6.0$ and submitted to $95^{\circ} \mathrm{C}$ for $30 \mathrm{~min}$ to antigenic recovery. Next, the blockade of endogenous peroxidase with hydrogen peroxide at $3 \%$ for $40 \mathrm{~min}$ was performed. Primary antibodies were applied and incubated for $20 \mathrm{~h}$ in a damp chamber at $4{ }^{\circ} \mathrm{C}$. After three more rinses in Buffered Saline Solution (PBS), secondary antibody (N-Histofine ${ }^{\circledR}$ ) was applied and incubated for $30 \mathrm{~min}$ at room temperature $\left(24^{\circ} \mathrm{C}\right)$. IHC reaction was revealed with DAB (Chromogen/Substrate Bulk Pack, ScyTek Laboratories) for $2 \mathrm{~min}$. In the negative control, the primary antibody was omitted and all slides were counterstained with hematoxylin.

All tissue photomicrographs for histological, histomorphometrical and IHC analyses were made under an optical microscope (LABOMED ${ }^{\circledR} \mathrm{LxPol}$ ) equipped with an Axio CAM HRc camera and Software Capture Pro 2.9.0.1.

\section{Data analysis}

Qualitative assessments of histological, histochemical and IHC of muscle samples were analyzed by observing three sections from each one of the animals $(n=8) /$ per group $=5$ : T0, T4 $m d x$-T and $m d x$-NT, T8 $m d x$-T an $m d x$-NT. The descriptive statistical analysis was performed via mean and standard error calculations (grip strength, Rotarod and intramuscular collagen fiber quantification). Analysis of the normality of the data was performed by the Shapiro-Wilk test and considering normal distribution the $\mathrm{p}$ value $>0.05$. To detect difference between groups the Student $t$ test analysis was conducted and to detect difference intra groups an Analysis of Variance (ANOVA) was performed and the Tukey test was used as post-hoc. The effect size (Cohen's d) was measured by Cohen's D Test. The IBM SPSS Statistics ver. 22.0 (IBM Co., Armonk, NY, USA) was used with the level of significance set at $\mathrm{p}<0.05$.

\section{RESULTS}

The $m d x$-T and $m d x$-NT groups presented an age dependent increased in corporal mass, without difference between groups. All $m d x$-T animals from Short (4 weeks) and Long Protocol ( 8 weeks) have finished the established treadmill protocol completing the $30 \mathrm{~min} / \mathrm{session}$ at $9 \mathrm{~m} / \mathrm{min}$. The mean values of the length of stay at Rotarod also have not shown difference between or intra groups (figure 2 a).

The normalized grip strength data are shown at figure $\mathbf{2} \mathbf{b}-\mathbf{d}$. There was a difference in T2 between groups $(\mathrm{p}=0.028$ ), with the $m d x$-T group presenting higher values at this time (figure $\mathbf{2} \mathbf{b}$ ). There was a decrease of the grip strength values at $\mathrm{T} 4$ related to the protocol training. The $m d x$-T showed difference between T2 to T4 ( $\mathrm{p}=0.009)$, T4 to T6 $(\mathrm{p}=0.022)$ and $\mathrm{T} 4$ to $\mathrm{T} 8(\mathrm{p}=0.002)$ (figure $2 \mathrm{D})$. There was an increase of grip strength of the trained animals after the $4^{\text {th }}$ week (T4) of training until the $\mathrm{T} 8$.

The $m d x$-NT group showed a gradual decreased on grip strength values until $\mathrm{T} 6$ and a slightly increase at T8 not superior to T0. There was significant difference between T0 to T2 $(\mathrm{p}=0.006)$, T0 to T4 $(\mathrm{p}=0.049)$, T0 to T6 $(\mathrm{p}=0.002)$, T0 to T8 $(\mathrm{p}=0.043), \mathrm{T} 4$ to T8 $(\mathrm{p}=0.047)$, and T6 to T8 $(\mathrm{p}=0.047)$ times of assessment (figure $2 \mathrm{c}$ ).

The area of intramuscular collagen fibers of SOL muscle was different between groups $(\mathrm{p}=0.025)$. At T4 $(0.31 \pm 0.04)$ it was lower than T0 to $m d x$-T animals $(0.48 \pm 0.05)(\mathrm{p}=0.04)$ (figure 3 a). The TA muscle showed different percentage of area between groups $(\mathrm{p}=0.002)$. The $m d x$-NT group at T8 $(0.31 \pm 0.03)$ was different from the $m d x$-T group at $\mathrm{T} 4(0.29 \pm 0.01)(\mathrm{p}=0.021)$ and T8 $(0.31 \pm 0.04)(\mathrm{p}=0.001)$ (figure $3 \mathbf{b}$ ).

For more details of Grip Strength, Rotarod and Intramuscular collagen deposition morphometry analysis, see the Supplementary material.

Typical histopathological features of dystrophic skeletal muscle were observed at SOL and TA muscle of both groups. Collagen fibers were distributed between muscular fibers with thicker tracts at perimysium than at endomysium. The $m d x$-T group presented innumerous thinner tracts at endomysium, mainly at $\mathrm{T} 4$ with thicker tracts observed at T0 than at T8, time were the collagen fibers had covered a higher area. Tracts of $m d x$-NT group were thicker at perimysium at $\mathrm{T} 0$ when compared to T4 (figure 4).

TA muscle showed thinner collagen fibers at perimysium of $m d x$-T group with the thicker ones at the perimysium of $m d x$-NT group on all assessed moments, especially at T8 (figure 5). 

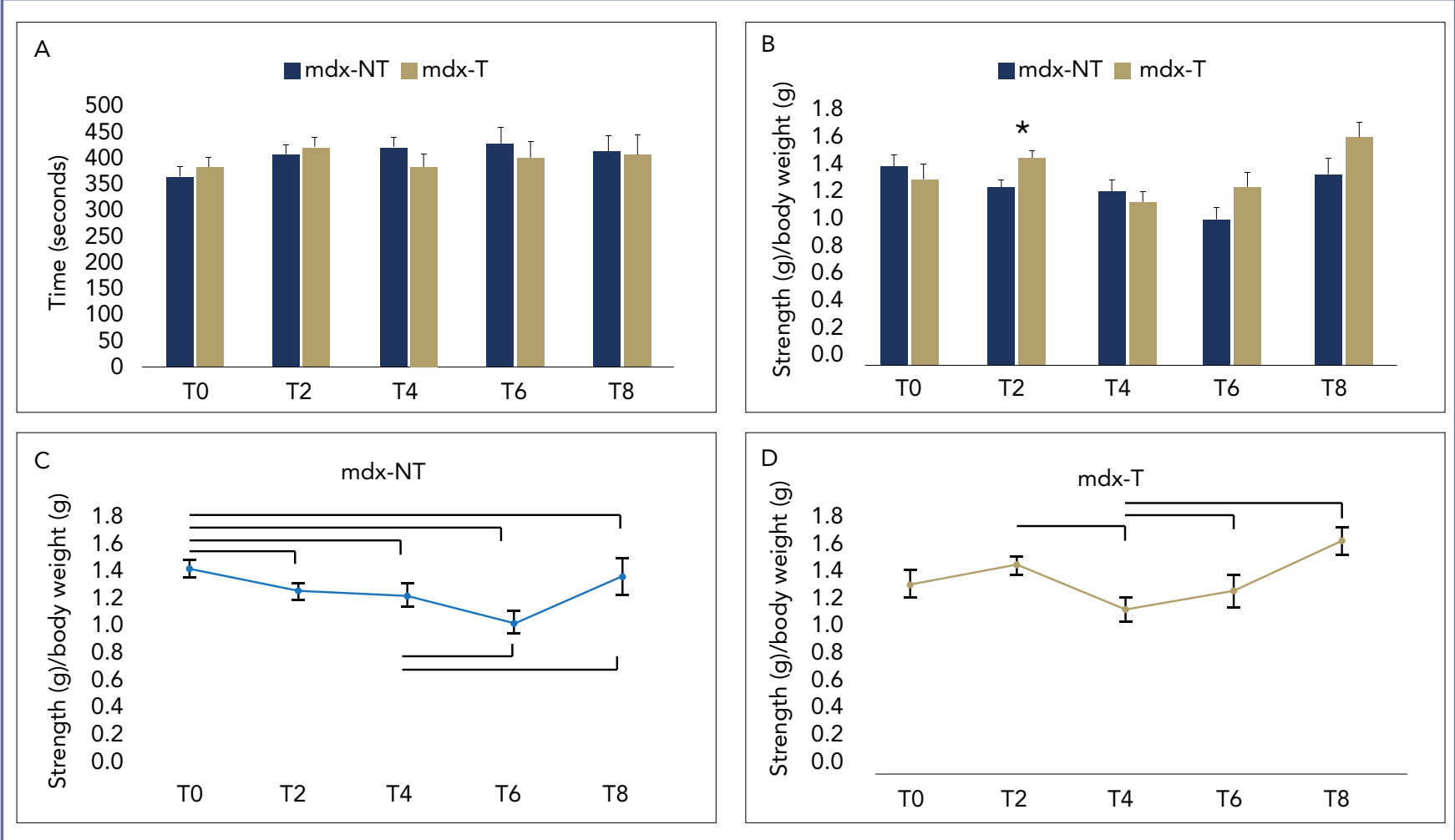

Figure 2. (A) Lenght of stay (seconds) at Rotarod over time of protocol training. (B) Normalized Grip Strength over time comparing $m d x-T$ and $m d x-N T$ groups. (C, D) Normalized Grip Strength of $m d x-N T$ and $m d x-T$. *Difference between groups, independent $t$ Test; horizontal bars show difference intra-group, paired t test with $p<0.05$.

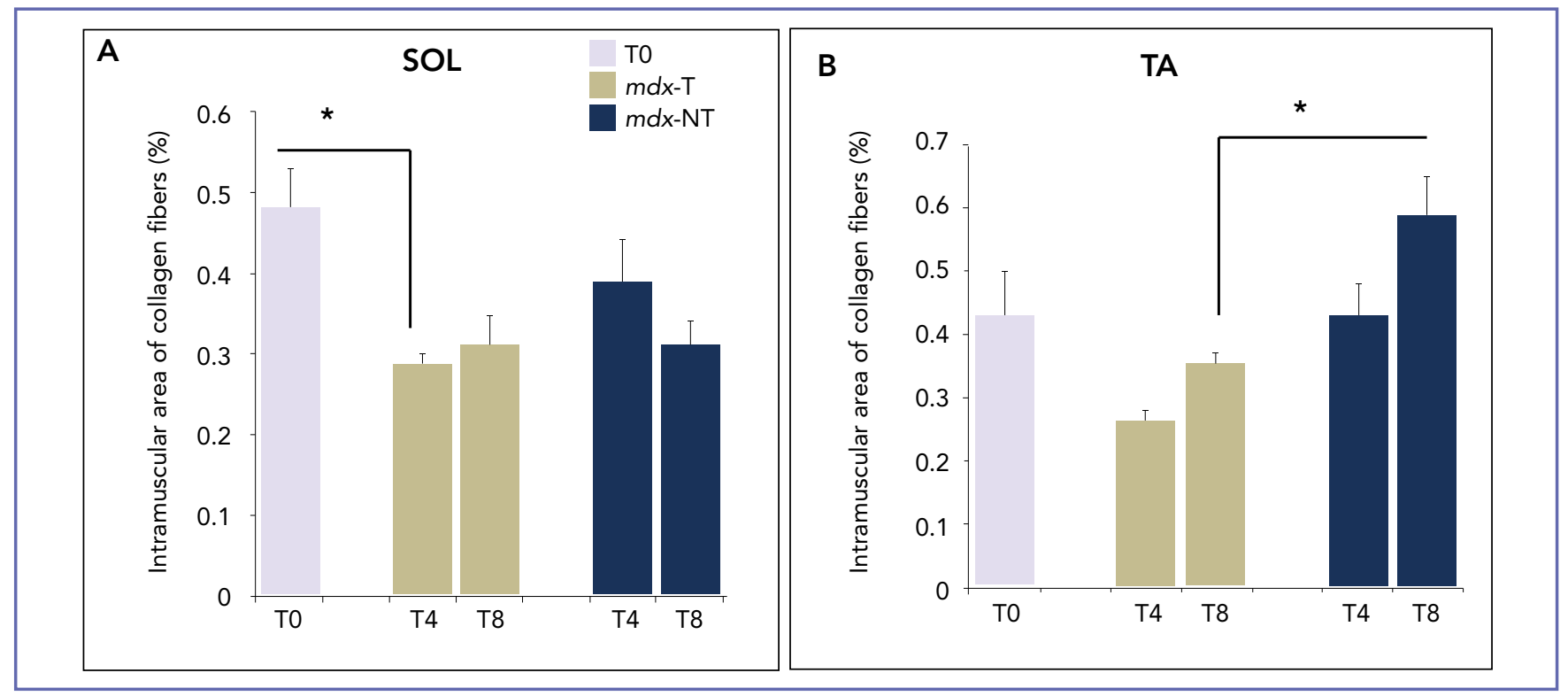

Figure 3. Effect of low-intensity training on the percentage of intramuscular collagen fibers deposition. Morphometric analysis of the percentage of intramuscular collagen fiber deposition in soleus (A) and tibial anterior (B). *Significant difference between groups (ANOVA with post-hoc Tukey). 


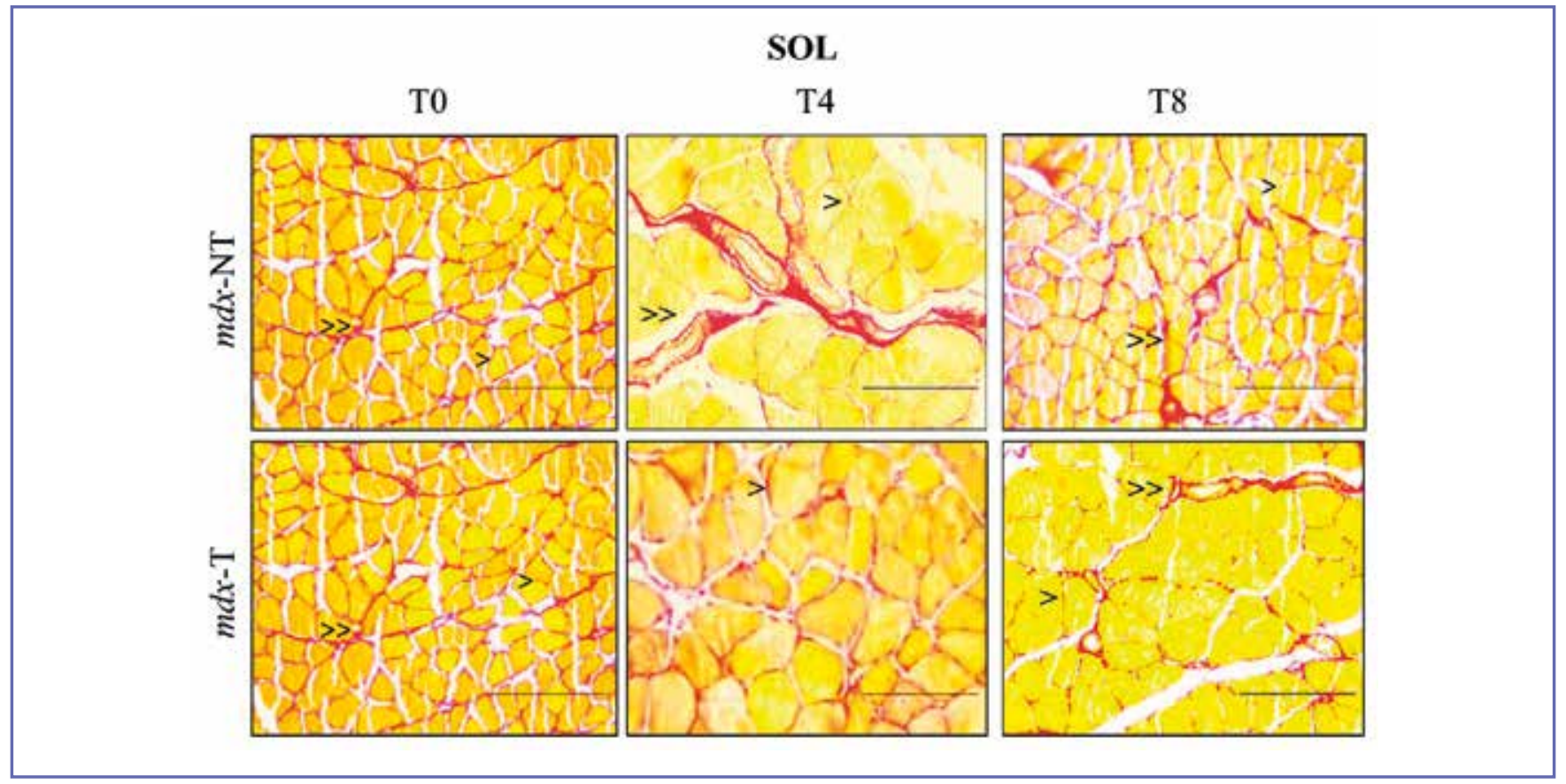

Figure 4. Morphological analysis of the deposition of collagen fibers on soleus muscle of $m d x-N T$ and $m d x-T$. Picrossirius red reaction. $>>$ Deposition of collagen fibers in the perimysium. $>$ Deposition of collagen fibers in endomysium. Scale bar $=100 \mu m$.

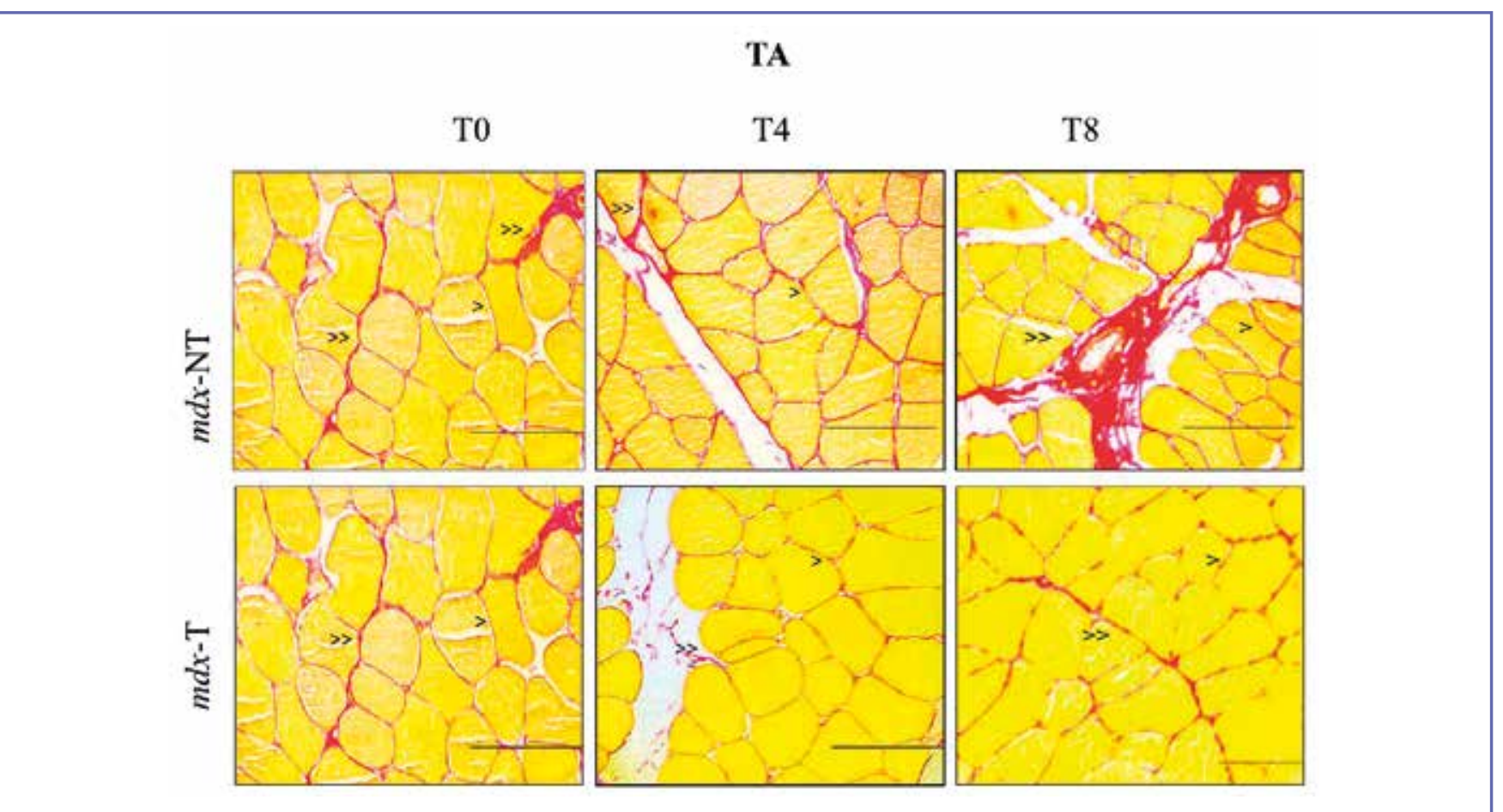

Figure 5. Morphological analysis of the deposition of collagen fibers on tibial anterior muscle of $m d x-N T$ and $m d x-T$. Picrossirius red reaction. >> Deposition of collagen fibers in the perimysium. > Deposition of collagen fibers in endomysium. Scale bar $=100 \mu \mathrm{m}$. 
Immunolocalization of TGF- $\beta 1$ has shown the presence of this pro fibrotic marker in the endomysium and perimysium of the TA and SOL muscles at T0, T4 and T8. It was also observed in sarcoplasm at T0 and $m d x$-NT at T4 and T8 (figure 6,7 ).

\section{DISCUSSION}

The low-intensity protocol training had maintained grip strength, performance on rotarod and favored a decline on the intramuscular collagen fibers area of tibial anterior muscle over time.

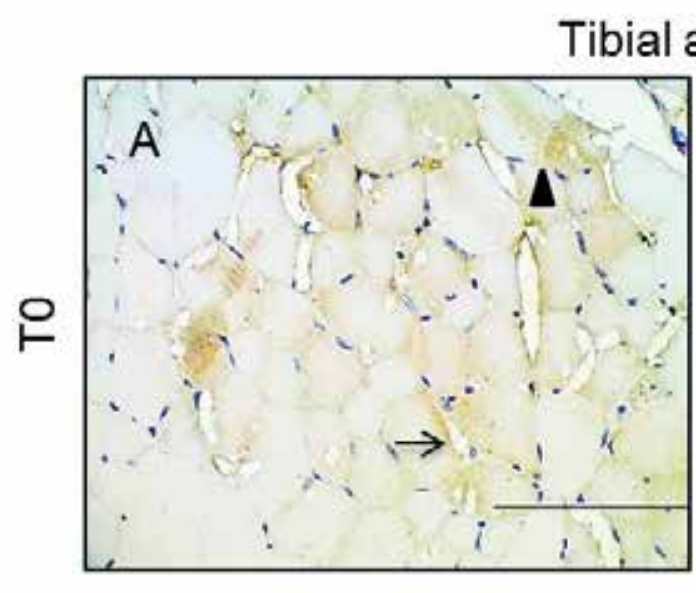

anterior
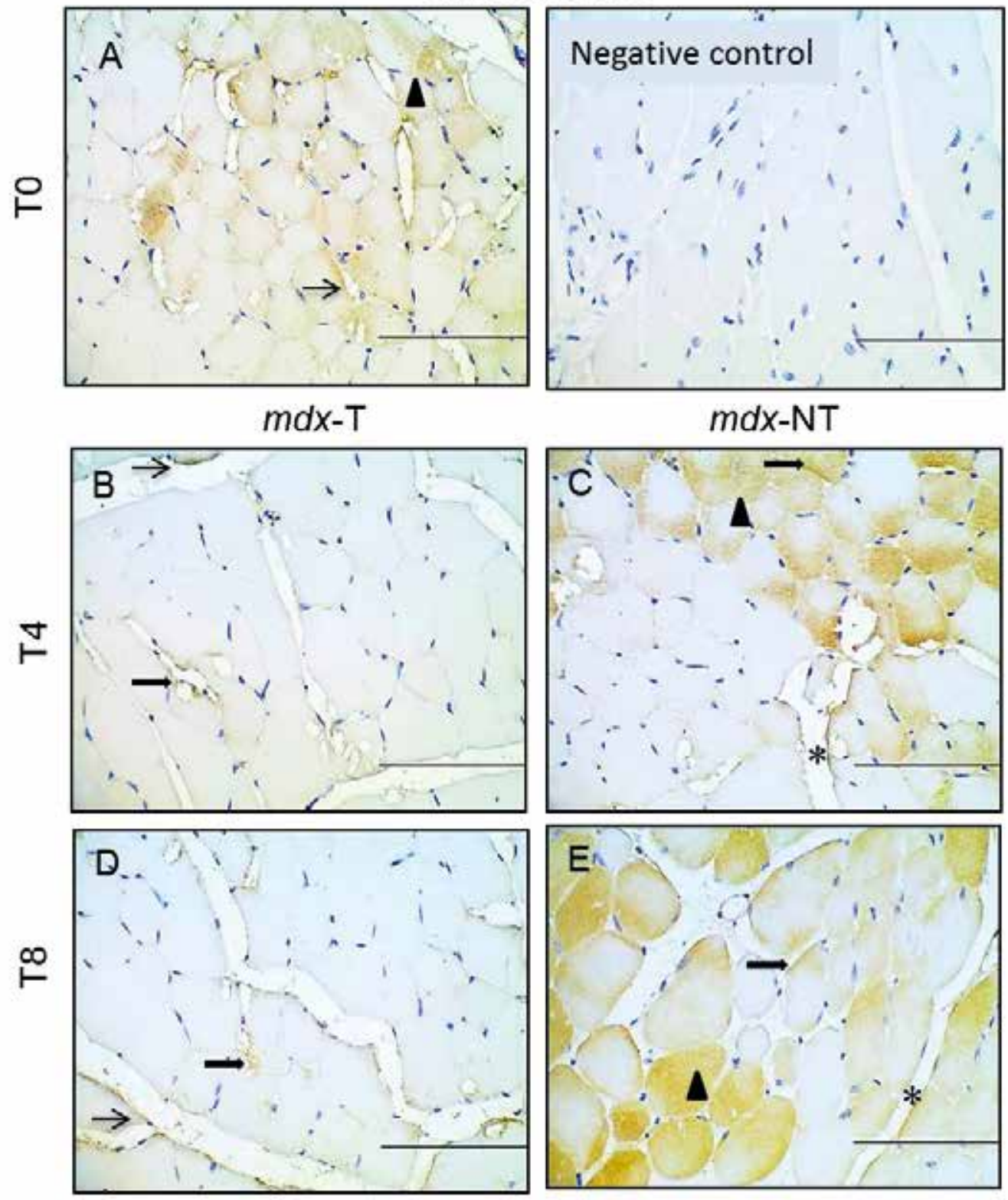

Figure 6. Morphological analysis of tibial anterior muscle of $m d x-T$ and $m d x-N T$ groups at T0, T4 and T8, 400x, IHC. Immunolocalization of TGF- $\beta 1$ and negative control of immunohistochemical reaction. On A: Tibial anterior muscle at T0; B $(m d x-T)$ and $\mathbf{C}(m d x-N T)$ at T4; D $(m d x-T)$ and $\mathbf{E}(m d x-N T)$ at T8. ${ }^{*}$ Perimysium $\rightarrow$ endomysium, $\rightarrow \boldsymbol{\Delta}$ sarcoplasm. Bar: $100 \mu m$. 


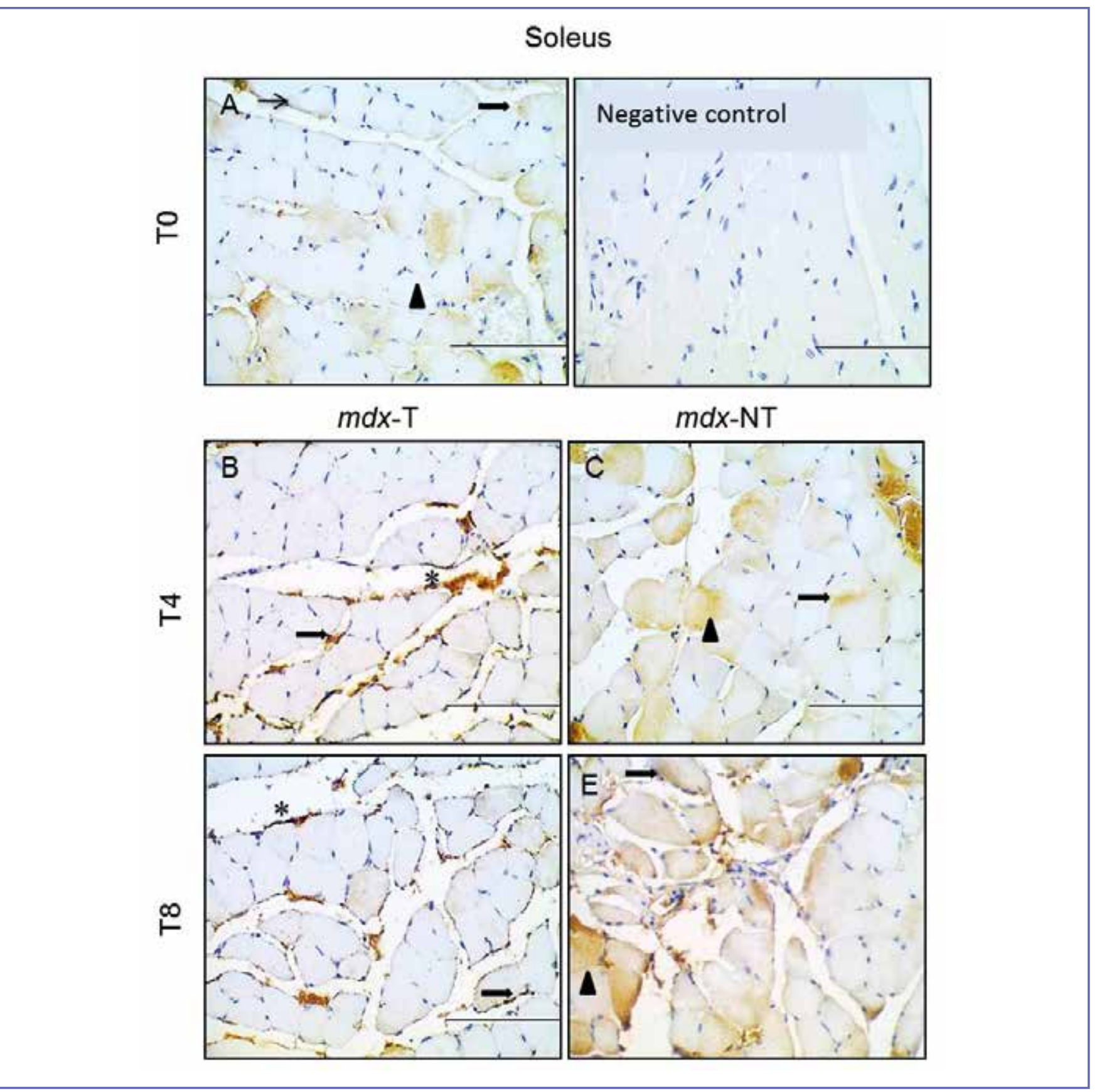

Figure 7. Morphological analysis of Soleus muscle of $m d x-T$ and $m d x-N T$ groups at T0, T4 and T8, 400x, IHC. Immunolocalization of TGF- $\beta 1$ and negative control of immunohistochemical reaction. On A: Soleus muscle at T0; B (mdx-T) and C (mdx-NT) at T4; $\mathrm{D}(\mathrm{mdx}-\mathrm{T})$ and $\mathrm{E}(\mathrm{mdx}-\mathrm{NT})$ at T8. ${ }^{\star}$ Perimysium $\rightarrow$ endomysium, $\rightarrow \boldsymbol{\Delta}$ sarcoplasm. Bar: $100 \mu \mathrm{m}$.

TGF- $\beta 1$ stained at endomysium and perimysium tracts of muscle fibers, as expected to dystrophic muscle. Sarcoplasm of sedentary animals was also stained to TGF- $\beta 1$ at T8. According to Ismaeel et al. (4) the expression of TGF- $\beta 1$ increase progressively with the progression of the disease and is positively correlated to the collagen density.

The effect of the intensity and of the time of treadmill training protocol on forelimb strength in vivo and on corporal 
mass of $m d x$ mice has been already studied (11). Our results attest that a training of low-intensity did not worsened muscle function of $m d x$ mice. It is interesting to note that at the moment were animals were 12 weeks old (at T4), the length of stay at Rotarod was lower than at the beginning or end of the 8 weeks of protocol. The same was observed to grip strength which suffered a decline at $\mathrm{T} 4$.

Some studies suggest that the strength of $m d x$ mice decrease after 3 months of age (15). It is related to degeneration/ regeneration cycles that are present between 4 and 15 weeks of age of these animals. This result is in accordance with the forelimb strength decrease that was found in $m d x$ mice of 5 to 12 weeks age that have not made other functional tests besides grip strength or treadmill running (18). Capogrosso et al. (19) submitted animals of 4 and 5 weeks age to a protocol of 12 weeks of treadmill training, at $12 \mathrm{~m} / \mathrm{min}$ and also found a decline on grip strength after 4 weeks of protocol. Even that trained and not trained mice have not shown difference between their grip strength (inter group), there was a considered increase of grip strength of trained mice between $\mathrm{T} 6$ to $\mathrm{T} 8$ that attest an adaptation to trained that could not be observed on sedentary ones.

Exercise regulates function, morphology and metabolism of skeletal muscle through modulation of different signaling pathways (11). Also, exercise decrease dysfunctional adipose tissue and improve oxygenation (20).

Physical exercise has been proposed as an adjuvant therapy to humans DMD aiming to maintain muscle strength and prevent contractures as long as possible. However, exercise practice remains controversial once dystrophic muscle can be damage due to excessive use (8). According to recent systematic reviews, besides some studies have demonstrate the benefice of exercise in slow up motor function damage in young people with $\mathrm{DMD}$, more research must be conducted to understand cardiac and respiratory function to exercise, the effect of long-term training protocol on dystrophic muscle and the effects of running and swimming on $\operatorname{DMD}(8,12)$.

The intensity of training and age of dystrophic animals influence the effects of exercise on dystrophic muscle. High intensity training using forced treadmill or downhill running causes loss of muscle strength, myofibres necrosis (21) and fibrosis (22).

Moreover, damage-related genes as the transforming growth factor- $\beta 1$ (TGF- $\beta 1$ ), tumor necrosis factor (TNF- $\alpha$ ) and tyrosine kinase c-src are severely increased, which reinforce lesion signs and muscle dysfunction (11).

Muscles are susceptible different to damage induced by exercise. Hindlimb muscles are directly involved in running exercises (19). Soleus and tibial anterior muscle are hindlimb muscles and are constituted by different fibers types which explain their different answer to training. In general, tibial anterior muscle is more affected to treadmill training because it has predominantly type II fibers (23). The fibrosis of tibial anterior muscle was influenced by low-intensity training protocol demonstrating reduce values at the end of the protocol in TA muscles of the animals that were submitted to exercise. We also observed a reduction of collagen fibers on soleus muscle after 4 weeks of protocol. Even in the dystrophic genotype, a muscle of slow type fibers is more resistant to mechanical challenge (11).

When fibrosis occurs, collagen not only increases its amount but also suffers post transduction dysfunction that alters its organization and contribute to tissue stiffness (18). Picrossirius red staining is one of the most known histochemical techniques able to selectively detect tracts of collagen and it becomes more specific when associated to the detection under polarized light (24).

On $m d x$ model the infiltration of immune cells reaches a peak between 4 and 8 weeks of age (9) and fibrosis can be seen after 10 weeks age (8), which is in accordance to our finding on the muscle of $m d x$ mice at T0 (8 weeks of age). In this way, we suggest that training had positively mediated collagen deposition in both studied muscle at T4 and had also kept smaller areas of fibrosis on trained mice in relation to sedentary $m d x$ mice.

Fibrosis was here studied by intramuscular collagen fibers morphometry using picrossirius red under polarized light and the immunolocalization of TGF- $\beta 1$. It is possible to observe that TGF- $\beta 1$ on $m d x$ mice skeletal muscle is age dependent and is located at epimysium and perimysium. Also, at sarcoplasm and around blood vessels of sedentary animals, features very close to intramuscular collagen fibers. Sedentary $m d x$ mice showed TGF- $\beta 1$ localized at sarcoplasm of muscle fibers, mainly at $\mathrm{T} 8$, time where they were 16 weeks old. TGF- $\beta 1$ is generally present at epimysium and perimysium of dystrophic muscle while on healthy muscle no stain can be seen to this pro fibrotic marker (25).

Song et al. (1) attested that the expression of TGF- $\beta 1$ is correlated to the degree of pathology and clinic progression of DMD. Its expression is regulated specifically at sarcoplasm of muscle cells and at myenteric plexus of human with DMD. Diverse animals' studies observed answers of TGF- $\beta$ to muscle damage by eccentric muscle contraction, a modality of contraction proven harmful to the dystrophic muscle (15). Studies show that levels of TGF- $\beta$ are increased after an eccentric muscle contraction (3).

\section{CONCLUSIONS}

As fibrosis reflects the final phase of a chronic inflammatory process typical of the dystrophic muscle, we suggest that low-intensity training can mediate the regeneration of 
muscle fibers of the hindlimb maintaining muscle strength during a chronic protocol of exercise. The limitation of this study is that cardiac and diaphragm muscles were not analyzed and the profibrotic marker was not quantified. The clinical contribution of these results is that DMD boys in the ambulatory phase of the disease can benefit of training considered of low-intensity. Further clinical studies are necessary to attest modalities of low-intensity training in humans at this phase and its real effects not only on clinical parameters but also on cardiac and respiratory muscle and limb muscles involved on functional activities.

\section{ACKOWLEDGMENTS}

This research was supported by $\mathrm{CNPq}$ (Nacional Council for Scientific and Technological Development) process number 421403/2016-0.

\section{CONFLICT OF INTERESTS}

The authors declare that they have no conflict of interests.

\section{REFERENCES}

1. Song Y, Yao S, Liu Y, et al. Expression levels of TGF- $\beta 1$ and CTGF are associated with the severity of duchenne muscular dystrophy. Exp Ther Med 2017;13(4):1209-14.

2. Grange RW, Gainer TG, Marschner KM, et al. Fast-twitch skeletal muscles of dystrophic mouse pups are resistant to injury from acute mechanical stress. Am J Physiol - Cell Physiol 2002;283(4 52-4):1090-101.

3. Kim J, Lee J. Role of transforming growth factor- $\beta$ in muscle damage and regeneration: Focused on eccentric muscle contraction. J Exerc Rehabil 2017;13(6):621-6.

4. Ismaeel A, Kim JS, Kirk JS, et al. Role of transforming growth factor- $\beta$ in skeletal muscle fibrosis: A review. Int J Mol Sci 2019;20(10).

5. Nakamura A, Takeda S. Mammalian models of duchenne muscular dystrophy: Pathological characteristics and therapeutic applications. J Biomed Biotechnol 2011;2011.

6. Serrano AL, Muñoz-Cánoves P. Fibrosis development in early-onset muscular dystrophies: Mechanisms and translational implications. Semin Cell Dev Biol 2017;64:181-90.

7. Thakur SS, Swiderski K, Ryall JG, Lynch GS. Therapeutic potential of heat shock protein induction for muscular dystrophy and other muscle wasting conditions. Philos Trans R Soc B Biol Sci 2018;373(1738).

8. Hyzewicz J, Ruegg UT, Takeda S. Comparison of Experimental Protocols of Physical Exercise for $m d x$ Mice and Duchenne Muscular Dystrophy Patients. J Neuromuscul Dis 2015;2(4):325-42.

9. Hyzewicz J, Tanihata J, Kuraoka M, et al. Low intensity training of $m d x$ mice reduces carbonylation and increas- es expression levels of proteins involved in energy metabolism and muscle contraction. Free Radic Biol Med 2015;82:122-36.

10. Zelikovich AS, Quattrocelli M, Salamone IM, et al. Moderate exercise improves function and increases adiponectin in the $m d x$ mouse model of muscular dystrophy. Sci Rep 2019;9(1):1-10.

11. Camerino GM, Cannone M, Giustino A, et al. Gene expression in $m d x$ mouse muscle in relation to age and exercise: Aberrant mechanical-metabolic coupling and implications for pre-clinical studies in Duchenne muscular dystrophy. Hum Mol Genet 2014:23(21):5720-32.

12. Kostek MC, Gordon B. Exercise Is an Adjuvant to Contemporary Dystrophy Treatments. Exerc Sport Sci Rev 2018;46(1):34-41.

13. Yiu EM, Kornberg AJ. Duchenne muscular dystrophy. J Paediatr Child Health 2015;51(8):759-64.

14. Padulo J, Oliva F, Frizziero A, Maffulli N. Muscles, ligaments and tendons journal - Basic principles and recommendations in clinical and field science research: 2016 update. MLTJ 2016;6(1):1-5.

15. Grounds MD, Radley HG, Lynch GS, Nagaraju K, De Luca A. Towards developing standard operating procedures for pre-clinical testing in the $\mathrm{mdx}$ mouse model of Duchenne muscular dystrophy. Neurobiol Dis 2008;31(1):1-19.

16. Grange RW. DMD_M.2.1.003: Use of treadmill and wheel exercise to assess dystrophic state. TREAT-NMD Neuromuscul Netw 2015;(Id):1-12.

17. Aartsma-Rus A, van Putten M. Assessing functional performance in the Mdx mouse model J Vis Exp 2014;(85):1-11.

18. Smith LR, Barton ER. Collagen content does not alter the passive mechanical properties of fibrotic skeletal muscle in $m d x$ mice. Am J Physiol - Cell Physiol 2014;306(10).

19. Capogrosso RF, Mantuano P, Cozzoli A, et al. Contractile efficiency of dystrophic $m d x$ mouse muscle: In vivo and ex vivo assessment of adaptation to exercise of functional end points. J Appl Physiol 2017;122(4):828-43.

20. Nieman DC, Wentz LM. The compelling link between physical activity and the body's defense system. J Sport Heal Sci 2019;8(3):201-17. Available from: https://doi.org/10.1016/j. jshs.2018.09.009.

21. Terrill JR, Radley-Crabb HG, Grounds MD, Arthur PG. $\mathrm{N}$-Acetylcysteine treatment of dystrophic $m d x$ mice results in protein thiol modifications and inhibition of exercise induced myofibre necrosis. Neuromuscul Disord 2012;22(5):427-34.

22. Nakamura A, Yoshida K, Takeda S, et al. Progression of dystrophic features and activation of mitogen-activated protein kinases and calcineurin by physical exercise, in hearts of $m d x$ mice. FEBS Lett 2002;520(1-3):18-24.

23. Staron RS, Kraemer WJ, Hikida RS, et al. Campos GER. Fiber type composition of four hindlimb muscles of adult Fisher 344 rats. Histochem Cell Biol 1999;111(2):117-23.

24. Rittié L. Method for Picrosirius Red-Polarization Detection of Collagen Fibers in Tissue Sections. In: Methods in Molecular Biology, Humana Press, New York, NY 2017.

25. Bernasconi P, Torchiana E, Confalonieri P, et al. Expression of transforming growth factor- $\beta 1$ in dystrophic patient muscles correlates with fibrosis. Pathogenetic role of a fibrogenic cytokine. J Clin Invest 1995;96(2):1137-44. 